\title{
IMPLICACIÓN DEL ALUMNADO EN LA EVALUACIÓN A TRAVÉS DE PORTFOLIO ELECTRÓNICO
}

Implication of the student in evaluation through electronic portfolio

Alunos na avaliação incidência através de carteira eletrônica

\author{
Vanesa Ausín Villaverde \\ Víctor Abella García \\ Vanesa Delgado Benito
}

(1) Universidad de Burgos, España. Teléfono: +34 947111418.Correo electrónico: vausin@ubu.es

(2) Universidad de Burgos, España. Correo electrónico: vabella@ubu.es

(3) Universidad de Burgos, España. Correo electrónico: vdelgado@ubu.es

\section{Resumen}

Esta comunicación pretende dar a conocer una experiencia de evaluación formativa y compartida llevada a cabo con alumnado de primer curso del Grado en Educación Infantil dentro de la asignatura “Tecnologías de la Información y Comunicación aplicadas a la Educación”. Se ha desarrollado durante el curso académico 2015/16 en la Universidad de Burgos y en ella participaron 64 estudiantes. El proceso de evaluación formativa se realizó a partir de la elaboración de un blog como portfolio de la asignatura. La actividad se evaluó a través de una rúbrica utilizada por el profesorado y por los estudiantes (autoevaluación y coevaluación). Paralelamente, se realizó evaluación formativa por parte de profesor durante todo el semestre y se realizó un seguimiento del funcionamiento de los grupos de trabajo.

Palabras clave: Evaluación formativa; rúbrica; portfolio; educación superior; evaluación compartida

\begin{abstract}
This communication is expected to show an experience in training and shared assessment. It was done by 64 students of the first course of early childhood education degree in the subject "Information and Communication Technologies applied to


Education". It was developed for the 2015/16 academic year at Burgos University and it consisted of creating a blog as the subject portfolio. The activity was assessed from teachers and students by using a rubric (self-assessment and co-evaluation). At the same time, a training assessment was done by the teacher for the whole semester, working checking the workgroup working.

Keywords: Formative assessment; rubric; portfolio; higher education; shared assessment

\section{Resumo}

Este trabalho tem como objectivo proporcionar uma experiência de avaliação formativa e compartilhada realizado com alunos do primeiro ano da Licenciatura em Educação Infantil dentro do tema "Tecnologias da Informação e Comunicação aplicadas à Educação". Ele foi desenvolvido durante o ano lectivo de 2015/16, na Universidade de Burgos e envolveu 64 alunos. O processo de avaliação formativa foi realizada a partir do desenvolvimento de um blog como uma carteira do assunto. A atividade foi avaliada usando uma rubrica usado por professores e alunos (auto-avaliação e de avaliação pelos pares). Da mesma forma, a avaliação formativa foi conduzido pelo professora o longo do semestre e monitorar o funcionamento dos grupos de trabalho foi realizada.

Palavras-chave: Avaliação formativa, rubrica, carteira, ensino superior, avaliação compartilhada

\section{Introducción}

En el contexto universitario actual, la participación activa del alumnado es un elemento clave en el transcurso de su formación académica, incluida su implicación en los procesos de evaluación.

La metodología utilizada por el docente y el sistema de evaluación asociado tienen una incidencia directa sobre el proceso de aprendizaje y la percepción que el estudiante tiene de las competencias adquiridas (Hortigüela, Pérez-Pueyo\& LópezPastor, 2015). En este sentido, entendemos la evaluación como un proceso sistemático orientado a unos fines claramente formativos y no enfocados únicamente a una calificación final, permitiendo al alumno conocer realmente el trabajo que lleva realizado y asesorarle sobre aquello que tiene que hacer para mejorarlo (Hortigüela, 
Abella, Delgado \& Ausín, 2016). A esta idea de evaluación formativa se une la importancia de contar con los estudiantes durante este proceso y que sean ellos partícipes del mismo, ya que como han demostrado diversas investigaciones, su participación en este proceso mejora su nivel de implicación y motivación en el proceso de enseñanza y aprendizaje (Brakke \& Brown, 2002; Ibarra \& Rodríguez, 2003).

El objetivo de esta comunicación es dar a conocer una experiencia de evaluación formativa y compartida a través de la utilización de rúbricas.

\section{Contextualización}

La experiencia se ha desarrollado en el Grado de Maestro de Educación Infantil de la Universidad de Burgos durante el curso 2015-2016, en la asignatura “TIC aplicadas a la educación”. Se trata de una materia de 6 créditos ECTS. El número de estudiantes matriculados asciende a 64, siendo 60 mujeres y 4 hombres.

\section{Diseño y desarrollo}

La actividad en la que se llevó a cabo la evaluación formativa y compartida consistió en la elaboración de un blog como portfolio del proyecto didáctico desarrollado durante el semestre y diseñado con la metodología de Aprendizaje Basado en Proyectos. El objetivo era que los estudiantes recogiesen las evidencias del trabajo que iban desarrollando durante el proyecto y las publicasen en el blog. La tarea se desarrolló en grupos de trabajo de 4 o 5 personas, teniendo un total de 16 grupos.

Al inicio se entregó la descripción detallada de la tarea, incluyendo los contenidos mínimos para que ésta pudiera ser evaluada, así como una rúbrica en la que se detallaban los criterios de evaluación del blog, siendo los alumnos conocedores de su implicación en la evaluación desde el inicio.

A lo largo del semestre el profesor fue revisando las entradas publicadas y diofeedback a los grupos de trabajo para reconocer los puntos fuertes y mostrarlos como buena práctica en clase y, por otro lado, hacer consciente al alumnado de los puntos débiles de su trabajo para poder mejorarlo a partir de los criterios de la rúbrica.

El proceso de seguimiento del trabajo también se desarrolló a través de la autoevaluación que realiza el propio grupo de su tarea y que luego se nutre con la información que recibe de la coevaluación de otro grupo de compañeros. Esto es, en dos 
momentos a lo largo del semestre los equipos revisan los blogs de otros grupos y seguidamente se reúnen para explicarse la evaluación, así como los puntos fuertes y débiles de los trabajos en base a la rúbrica.

Al finalizar el semestre los blogs fueron evaluados mediante un proceso de autoevaluación y coevaluación por otro equipo. De esta forma, cada blog recibió tres notas (la del profesor, la de su autoevaluación y la de la coevaluación). Conscientes de las dificultades que entraña este tipo de evaluación, el profesorado explicó a los estudiantes la importancia de llevar a cabo una evaluación seria y ajustada a la realidad a partir de los criterios recogidos en la rúbrica. En el caso de existir grandes discrepancias entre las notas asignadas, el profesorado sería el que otorgara la nota final, penalizando, restando un punto sobre la calificación final, al grupo que no hubiera realizado correctamente la evaluación.

\section{Evaluación}

Tras analizar las calificaciones finales de la actividad, obtenemos que las notas medias se encuentran en un 7,3. Por agentes de evaluación, tenemos que en las calificaciones otorgadas por el profesor la media se encuentra en un 7,4; la nota media de las autoevaluaciones asciende a un 7,6 y la media de la coevaluación es un 7,1. Solo en un $10 \%$ de los grupos de trabajo ha tenido que intervenir el equipo docente porque se había realizado una evaluación poco ajustada a la realidad de la rúbrica. En general, los estudiantes realizan bien las evaluaciones ya que al unificar las notas comprobamos que, en la mayoría de los casos, la diferencia no llega a un punto más o menos.

\section{Conclusiones}

A la vista de los resultados obtenidos, creemos que los procesos de evaluación formativa y compartida permiten al alumnado estar más implicados en la tarea que realizan, así como, el procedimiento que les ayuda a adquirir un mayor conocimiento de su propio proceso de evaluación. Como elemento clave en este proceso, destacamos el seguimiento continuo que se realiza por parte del docente y las autoevaluaciones y coevaluaciones que se desarrollan a lo largo del semestre, que facilita a los equipos detectar las debilidades de su trabajo y poder reflexionar sobre ellas. La rúbrica es el instrumento que nos permite articular este protocolo de evaluación formativa y compartida, proporcionando objetividad y trasparencia a este proceso. 


\section{Referencias}

Brakke, D. F., \& Brown, D. T. (2002).Assessment to improve student learning. New Directions for Higher Education, 119, 119-122.

Hortigüela, D., Abella, V., Delgado, V., \& Ausín, V. (2016). Influencia del sistema de evaluación empleado en la percepción del alumno sobre su aprendizaje y las competencias docentes. Infancia, Educación y Aprendizaje (IEYA), 2(1), 20-42

Hortigüela, D., Pérez-Pueyo, A., \& López-Pastor, V. (2015). Implicación y regulación del trabajo del alumnado en los sistemas de evaluación formativa en educación superior. Relieve: Revista Electrónica de Investigación y Evaluación Educativa, 21(1), 1-5.

Ibarra Sáiz, M. S., \& Rodríguez Gómez, G. (2003). El trabajo colaborativo en las aulas universitarias: reflexiones desde la autoevaluación. Revista de Educación, 344, 355-375. 